\title{
Sleep-Disordered Breathing, Quality of Sleep and Chronotype in a Cohort of Adult Patients with Bardet-Biedl Syndrome
}

\author{
Léa Dormegny (D) ${ }^{1,2}$ \\ Reana Velizarova (iD \\ Carmen M Schroder ${ }^{1,3}$ \\ Ulker Kilic-Huck ${ }^{1,3}$ \\ Henri Comtet ${ }^{1,3}$ \\ Hélène Dollfus ${ }^{4}$ \\ Patrice Bourgin 1,3 \\ Elisabeth Ruppert ${ }^{1,3}$ \\ 'Centre des Troubles du Sommeil - \\ CIRCSom, Hôpital Civil, University of \\ Strasbourg, Strasbourg, 6709I, France; \\ ${ }^{2}$ Department of Ophthalmology, Hôpital \\ Civil, University of Strasbourg, \\ Strasbourg, 6709I, France; ${ }^{3}$ Institute for \\ Cellular and Integrative Neurosciences, \\ CNRS - UPR 3212, Strasbourg, 67000, \\ France; ${ }^{4}$ Centre des Affections Rares en \\ Génétique Ophtalmologique (CARGO), \\ University of Strasbourg, Strasbourg, \\ 6709I, France
}

Objective/Background: Bardet-Biedl syndrome (BBS) is a rare but well-recognized ciliopathy with high genetic and phenotypic heterogeneity. Cardinal features include obesity, diabetes and high blood pressure (HBP), which are often associated with sleep-disordered breathing. Also, the high prevalence of blindness due to retinal dystrophy could affect circadian sleep-wake rhythms. We characterized in this cohort of adult BBS patients sleepdisordered breathing, sleep quality, daytime sleepiness and chronotype.

Patients and Methods: Thirty-two patients with genetically confirmed BBS were included in this observational single center study. Overnight respiratory polygraphy was performed for sleep apnea syndrome (SAS) in 30 patients. Quality of sleep, daytime sleepiness, fatigue and chronotype were assessed in 25 patients using Pittsburgh sleep quality index (PSQI), 14-day sleep diary (SD), Epworth sleepiness scale (ESS), Pichot fatigue scale (PFS) and Horne and Ostberg morningness-eveningness questionnaire (MEQ).

Results: Patients' mean age was $32 \pm 11$ years and mean BMI $32.6 \pm 7.7 \mathrm{~kg} / \mathrm{m}^{2}$. Eleven (35\%) patients had HBP and 7 (22\%) diabetes. Moderate to severe sleep apnea syndrome (SAS) was present in $5(17 \%)$ and was not associated with altered sleep, daytime sleepiness or fatigue. Most of the patients $(63 \%)$ evaluated their sleep as of good quality (PSQI $\leq 5$ ). Median scores of sleep quality, daytime sleepiness and fatigue were normal (PSQI of 3.0 [2.0-6.0], ESS of 9.0 [6.0-13.0] and PFS of 8.0 [3.0-13.0], respectively). Predominant chronotypes according to MEQ were either "intermediate" $(57 \%)$ or "moderate morning" (29\%). None had a free running sleep-wake cycle. 14-day SD revealed overall few awakenings at night and low daytime napping.

Conclusions: Given the cardiovascular risk factors, systematic screening for SAS should be considered in BBS patients, regardless of sleep and daytime vigilance complaints. None of these highly visually impaired patients had a circadian sleep-wake rhythm disorder. Further objective assessments are needed to better characterize sleep and circadian rhythms in BBS patients.

Keywords: Bardet-Biedl syndrome, ciliopathy, sleep disordered breathing, sleep apnea syndrome, chronotype, circadian rhythm sleep wake disorder

\section{Plain Language Summary}

Blindness, obesity, diabetes, high blood pressure and hypotonia are features of Bardet-Biedl syndrome (BBS) which might impede sleep quality. However, quality of sleep in BBS is poorly characterized. Blindness could lead to impaired circadian rhythms (ie, daily 24-hour rhythms driven by the internal circadian clock and mainly synchronized in the sighted by light dark cycles). Obesity, hypotonia, diabetes and high blood pressure might lead to sleep disordered breathing.
Correspondence: Elisabeth Ruppert Centre des Troubles du Sommeil CIRCSom - Hôpital Civil, I, place de l'Hôpital, BP 426, Cedex, Strasbourg, 6709I, France

Tel +33388 II 6430

Fax +33388 II 5153

Email elisabeth.ruppert@chru-strasbourg.fr 
We characterized nighttime respiration, quality of sleep, daytime sleepiness and chronotype using overnight respiratory polygraphy and validated assessment scales in 32 adult BBS patients.

Prevalence of moderate and severe sleep apnea syndrome (SAS) was high (17\%) given the age of our population (mean age $32 \pm 11$ years). SAS was not associated with altered sleep, daytime sleepiness or fatigue. Sleep quality and daytime vigilance were overall preserved. Intermediate and moderate morning chronotypes were predominant. No circadian rhythm sleepwake disorder or free-run (ie, circadian profile different than the 24-h day entrainment) were present according to sleep diaries.

Despite features predisposing to altered quality of sleep, daytime wakening and circadian rhythms, BBS patients declared overall satisfying sleep quality and normal daytime sleepiness.

Systematic screening for SAS should be considered in BBS patients, regardless of sleep and daytime vigilance complaints.

None of these highly visually impaired patients had a circadian sleep-wake rhythm disorder. Further objective assessments are needed to better characterize sleep and circadian rhythms in BBS patients.

\section{Introduction}

Bardet-Biedl syndrome (BBS) is a rare ciliopathy whose prevalence falls below $1 / 100,000$ in Europeans. ${ }^{1}$ Mutations in over twenty different genes involved in the development or function of primary cilia are related to BBS and even more remain to be described. ${ }^{2}$ Main clinical features reported are retinal dystrophy, postaxial limb defects, overweight, learning difficulties, renal tract abnormalities, hypogonadism and high blood pressure. Hypotonia, glucose intolerance, nephrogenic insipid diabetes and liver fibrosis are some of the minor features. Poor specificity along with slow development of those clinical features lead to delayed diagnosis at the average age of nine years. ${ }^{3}$ Blindness, chronic renal failure and diabetes are most invalidating conditions in adults. ${ }^{3-7}$ Most deleterious body damages caused by BBS are the cardiovascular complications related to diabetes, HBP, chronic renal failure and obesity and their incidence is growing with the patient's ageing.

Quality of sleep in BBS is poorly characterized. Only one very recent study reports sleep efficiency calculated from the ratio of total sleep duration to time in bed using 12-day wrist-worn accelerometer records in a large BBS population. Median ratio (25th, 75 th centiles) reaches $89.89(87.73,92.74)$ in adults, with a slightly higher ratio in children. ${ }^{8}$ Retinal dysfunction leading to complete blindness in BBS patients could impair light entrainment of the circadian clock. ${ }^{9,10}$ Moreover, obesity and hypotonia could promote sleep disordered breathing (SDB) and impair quality of sleep and daytime vigilance in BBS patients. ${ }^{11}$ This study aimed to characterize quality of sleep and vigilance, chronotype and breathing during the night in a cohort of adult BBS patients.

\section{Patients and Methods}

This single center observational study with nationwide recruitment included solely patients with a genetically confirmed diagnosis of BBS. Ethical approval had been received from CPP "EST IV" (Strasbourg, France) 10 June 2008 for the study PHRC National Bardet Biedl 2007 IDRCB 2007-A00868-45. Procedures used in this study adhered to the tenets of the Declaration of Helsinki. Written informed consent was obtained from all the patients or their legal representatives when necessary.

Thirty-two BBS patients were referred to our Sleep Center between August 2009 and January 2013. All underwent an overnight respiratory polygraphy (EMBLETTAC) Gold portable sleep apnea monitoring device; ResMed, Paris, France) with multiple sensors including heart rate, oxygen saturation $\left(\mathrm{SaO}_{2}\right)$ and thoracic and abdominal belts. One record was excluded because of insufficient quality, and one other due to intolerance to the device. All records were scored manually (B.K.) and the apneahypopnea index (AHI) calculated as the number of apnea and hypopnea episodes per hour of scoring time. ${ }^{12}$ Absence of sleep apnea syndrome (SAS) was defined by AHI $<5$, mild SAS by $5 \leq$ AHI $<15$, moderate SAS by 15 $\leq \mathrm{AHI}<30$ and severe SAS by $\mathrm{AHI} \geq 30$. The percentage of scoring time with $\left(\mathrm{SaO}_{2}\right)$ below $90 \%$ was reported.

Starting from January 2010, twenty-five patients (78\%) were further characterized for quality of sleep, daytime sleepiness, fatigue and chronotype using the Pittsburgh Sleep Quality Index (PSQI), a 14-day sleep diary (SD) including a daily numerical evaluation of sleep satisfaction (from 0 being worse to 10 being best), the Epworth Sleepiness Scale (ESS), the Pichot Fatigue Scale (PFS) and the Horne and Ostberg Morningness-Eveningness Questionnaire (MEQ). ${ }^{13-16}$ Due to participants' low visual acuity, an examiner read aloud questionnaires' items and reported the answers. Sleep efficacy (SE) was calculated from the ratio of total sleep duration to time in bed using data from both PSQI (SE-PSQI) and 14-day SD (SE-SD). For this parameter, score went from 0 (worst sleep efficacy) to 1 (best sleep efficacy). Nineteen patients out of the twenty-five had completed the entire 14-day SD. No systematical screening had been done for other sleep 
disorders. Consequent to the study, a complaint about sleep onset and maintenance insomnia was reported by two patients associated to restless legs syndrome that was severe in one and moderate in the other. One patient complained of a more complex hypersomnolence in a context of a long sleeper with insufficient sleep syndrome due to early wake-up times and a late chronotype.

Patients were examined for body mass index (BMI), diabetes and high blood pressure (HBP). All underwent spirometry with diluted helium method and ophthalmological examination including fundus examination and electroretinography. Best corrected visual acuity (BCVA) was assessed with $\log$ MAR scale. Central macular thickness (CMT) was measured on optical coherence tomography (OCT).

Patients presenting with a good quality of sleep (ie, PSQI $\leq 5)$ were compared to patients with a poor quality of sleep (ie, PSQI > 5) for AHI, percentage of scoring time with $\mathrm{SaO}_{2}$ below 90\%, BCVA, CMT, and MEQ using Student's t-test.

\section{Results}

\section{General Characteristics of the Population}

Thirty-two patients were included, 13 (41\%) males and 19 (59\%) females. Mean age was 32 \pm 11 [19-63] years. Eight different BBS genotypes were reported (BBS1, BBS4, BBS5, BBS7, BBS9, BBS10, BBS12 and BBS17). All patients had severe pigmentary retinopathy confirmed on fundus examination, visual field and electroretinography. Mean visual acuity was $1.86 \pm 0.91 \log \mathrm{MAR}$ on the right eye and $1.86 \pm 0.94 \log$ MAR on the left eye. One patient had no light perception in both eyes and one had light perception only in one eye. Mean CMT on OCT was 160.7 $\pm 43.4 \mu \mathrm{m}$ on the right eye and $148.1 \pm 41.1 \mu \mathrm{m}$ on the left eye.

Mean BMI was $32.6 \pm 7.7 \mathrm{~kg} / \mathrm{m}^{2}$ with 12 patients $(37 \%)$ being obese and 7 (22\%) having morbid obesity. High blood pressure was present in eleven $(35 \%)$ patients and type 2 diabetes in seven (22\%). Spirometry exam showed moderate restrictive ventilatory defect in 5/22 (22.7\%) patients and severe restrictive ventilatory defect in one. General characteristics of the patients are listed in Table 1.

\section{Sleep Disordered Breathing}

Thirty ventilatory polygraphy records were analyzed. While 20 patients presented with no SDB (mean age 30 \pm 10 years), 5 had mild SAS (mean age $33 \pm 12$ years). Moderate to severe SAS was diagnosed in 5 patients
(17\%) (mean age $45 \pm 10$ years). Four out of these latter five had obstructive SAS, comorbid high blood pressure (HBP) and either obesity (BMI $\left.\geq 30 \mathrm{~kg} / \mathrm{m}^{2}\right)(\mathrm{n}=2)$ or morbid obesity $\left(\mathrm{BMI} \geq 40 \mathrm{~kg} / \mathrm{m}^{2}\right)(\mathrm{n}=2)$. Two had type 2 diabetes (Table 1). Central apneas (index of 19/h) were present in a 44-year-old patient presenting with total AHI of $33 / \mathrm{h}$. The latter patient had normal BMI and no underlying etiology was identified for these apneas.

\section{Sleep Quality and Daytime Fatigue}

According to PSQI, 6 patients (25\%) reported a sleep latency of more than 30 minutes and $8(33 \%)$ reported at least 2 awakenings during the night. Median PSQI score was 3.0 [2.0-6.0], with most of the patients (63\%) evaluating their sleep as of good quality (as defined by PSQI $\leq 5$ ) (Table 1). Numerical evaluation of sleep quality was consistent with this first result (median score was 8.6/10 [7.4-9.9]). Median SE-PSQI was 1.0 [0.9-1.0] and 79\% of the patients had good SE-PSQI as defined by a ratio $\geq 0.85$. Median SE-SD was 0.98 [0.88-1.0] and $82.3 \%$ had a result $\geq 0.85$.

Daytime sleepiness evaluated by ESS was normal $(\leq 10 / 24)$ in 14 patients $(56 \%)$, moderate (ESS $\geq 11$ and $<18 / 24)$ in 9 patients $(36 \%)$ and severe (ESS $\geq 18 / 24)$ in 2 $(8 \%)$. Medians for ESS are exposed in Table 1. Fatigue as evaluated by PFS was normal $(\leq 10 / 24)$ in 16 patients $(64 \%)$, moderate (PFS $\geq 11$ and $\leq 20)$ in 8 patients $(32 \%)$ and severe (PFS $\geq 21$ ) in 1 patient $(4 \%)$. On their 14-day SD, $9(26 \%)$ patients reported to take a nap at least once a week and, from these 9, 5 reported this at least 3 times a week. Median daytime napping in these 9 patients was 60 [30-60] minutes.

\section{Chronotype Assessment}

According to MEQ, more than half of the patients $(57 \%)$, aged of $28 \pm 7$ years in mean, had an intermediate chronotype and one-third (29\%), aged of $48 \pm 11$ years in mean, had a moderate morning chronotype. Solely one patient aged 28 had a moderate evening chronotype, another patient also aged 28 had a definite evening chronotype and a definite morning chronotype was present in a patient aged 29 years old. According to their 14-day $\mathrm{SD}$, no patient presented with a circadian rhythm sleepwake disorder (CRSWD), especially none was freerunning. The patient without any light perception had a moderate morning chronotype and regular sleep wake cycles. She had severe obstructive SAS and no major complaint about sleep or daytime vigilance. 


\begin{tabular}{|c|c|c|c|c|c|c|c|c|c|c|c|c|c|c|c|c|c|c|c|c|c|}
\hline 0 & & $\triangleq$ & \begin{tabular}{l|}
$\frac{\Gamma}{y}$ \\
$\frac{1}{d}$ \\
0 \\
+1 \\
0 \\
0
\end{tabular} & 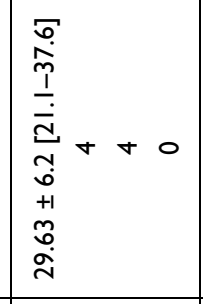 & 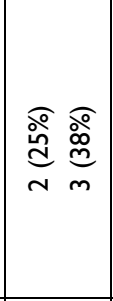 & $\underbrace{\stackrel{\widehat{్}}{\mathrm{O}}}_{\text {in }}+-0$ & $\begin{array}{l}\sigma \\
\dot{p} \\
\underline{1} \\
\dot{\vdots} \\
a \\
\underline{n}\end{array}$ & 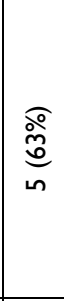 & $\underset{\substack{\stackrel{0}{0} \\
\stackrel{c}{N}}}{ }$ & 0 & 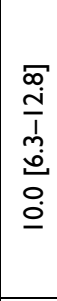 & 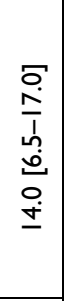 & ' & $\begin{array}{l}\sigma \\
0 \\
0 \\
0 \\
0 \\
0 \\
o \\
0\end{array}$ & $\begin{array}{l}\infty \\
0 \\
0 \\
\hat{0} \\
0 \\
\infty \\
0 \\
0\end{array}$ & 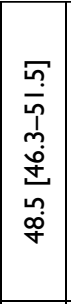 & 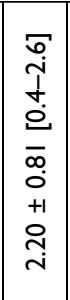 & 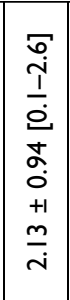 & 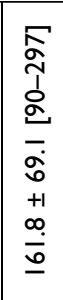 & 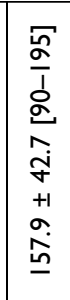 & 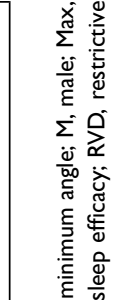 \\
\hline$z$ & $=$ & $\widehat{\hat{f}}$ & 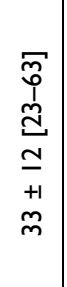 & 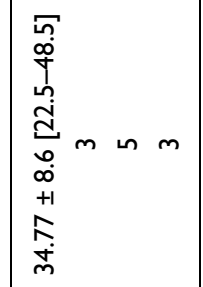 & 高高 & $\underbrace{\stackrel{\circ}{\mathrm{d}}}_{n} m--$ & $\begin{array}{l}\overrightarrow{0} \\
\stackrel{0}{0} \\
0 \\
\stackrel{0}{0} \\
\infty \\
\dot{i}\end{array}$ & 总 & $\stackrel{\circ}{\stackrel{\circ}{\varrho}}$ & 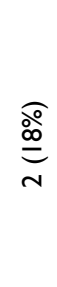 & ' & $\begin{array}{l}\tilde{n} \\
\stackrel{n}{n} \\
\vdots \\
0 \\
\dot{n} \\
0 \\
\infty \\
\infty\end{array}$ & 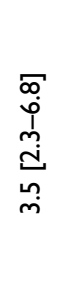 & 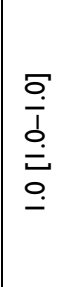 & $\begin{array}{l}\sigma \\
\dot{1} \\
\infty \\
0 \\
0 \\
o \\
0\end{array}$ & 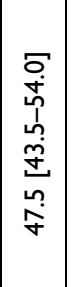 & $\begin{array}{l}0 \\
\dot{1} \\
\dot{j} \\
\dot{0} \\
o \\
0 \\
+1 \\
+1 \\
0 \\
\dot{j}\end{array}$ & 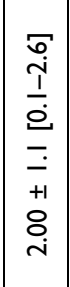 & 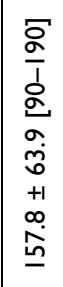 & 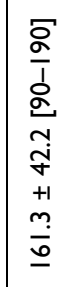 & 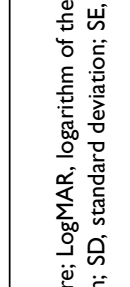 \\
\hline 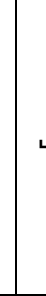 & n & 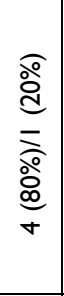 & 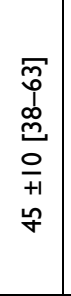 & 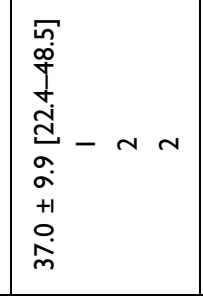 & 总 & $\underbrace{\stackrel{\circ}{\circ}}_{m}--$ & 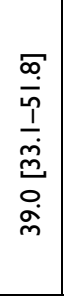 & & ' & & 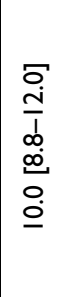 & 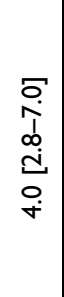 & 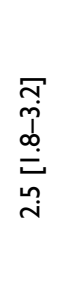 & 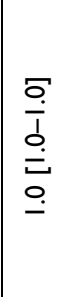 & $\begin{array}{l}\sigma \\
0 \\
0 \\
0 \\
0 \\
0 \\
0 \\
0\end{array}$ & 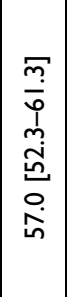 & 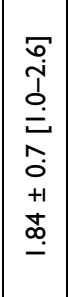 & 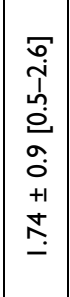 & 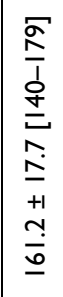 & 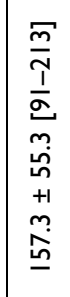 & 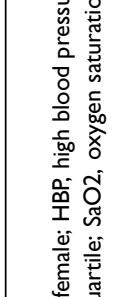 \\
\hline 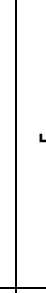 & n & 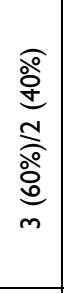 & $\begin{array}{l}\bar{n} \\
\mathfrak{p} \\
\stackrel{d}{d} \\
\simeq \\
+1 \\
m \\
m\end{array}$ & 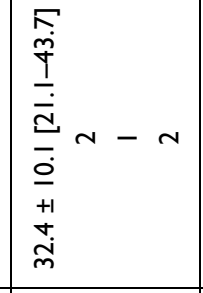 & 总 & $\underbrace{\stackrel{\Phi}{\circ}}_{\sigma}+00$ & 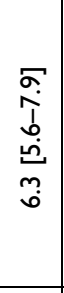 & & ' & & $\begin{array}{l}\sigma \\
0 \\
0 \\
0 \\
\dot{+} \\
0 \\
\dot{+}\end{array}$ & 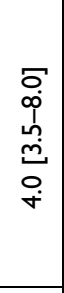 & 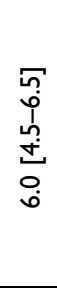 & $\begin{array}{l}\sigma \\
\vdots \\
\vdots \\
o \\
0 \\
o \\
0 \\
0\end{array}$ & $\begin{array}{l}\sigma \\
\frac{\sigma}{1} \\
0 \\
0 \\
0 \\
a \\
0\end{array}$ & 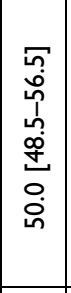 & 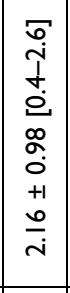 & 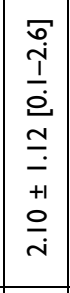 & 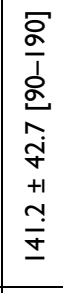 & 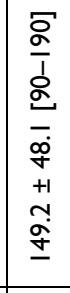 & 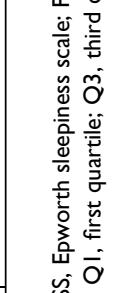 \\
\hline 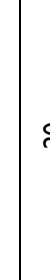 & $\vec{v}$ & 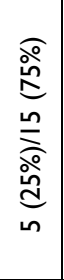 & $\begin{array}{l}\bar{\sigma} \\
o \\
0 \\
0 \\
0 \\
+1 \\
0 \\
0\end{array}$ & 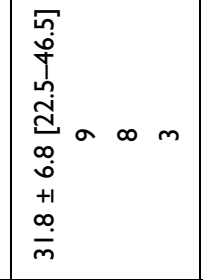 & 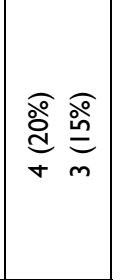 & 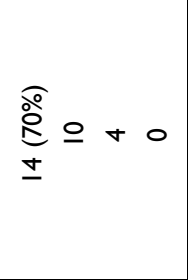 & $\begin{array}{l}\sigma \\
\stackrel{i}{i} \\
\underline{i} \\
\stackrel{n}{i}\end{array}$ & & , & & 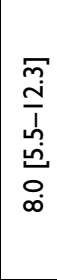 & $\begin{array}{l}\frac{n}{1} \\
\infty \\
\\
\stackrel{n}{n} \\
\end{array}$ & $\begin{array}{l}\square \\
0 \\
0 \\
0 \\
\dot{d} \\
0 \\
\dot{m}\end{array}$ & $\begin{array}{l}\sigma \\
\frac{0}{1} \\
0 \\
0 \\
0 \\
0 \\
0\end{array}$ & $\begin{array}{l}0 \\
\frac{\sigma}{1} \\
0 \\
0 \\
0 \\
0 \\
0\end{array}$ & 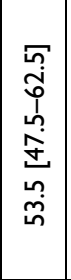 & 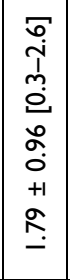 & 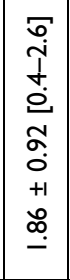 & 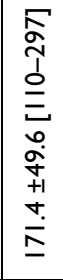 & 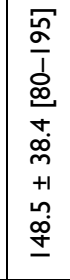 & 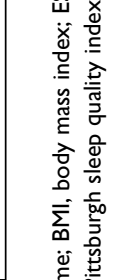 \\
\hline & nิ & 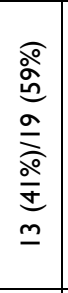 & 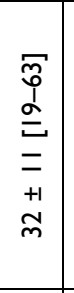 & 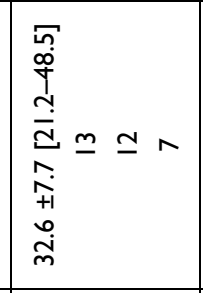 & 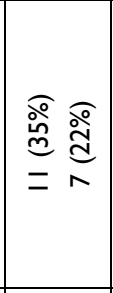 & 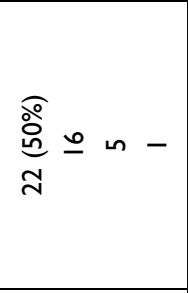 & $\begin{array}{l}\bar{a} \\
b \\
0 \\
\dot{\omega} \\
\bar{m}\end{array}$ & 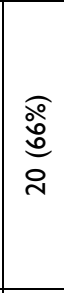 & 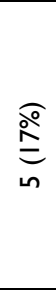 & $\underbrace{\stackrel{\overbrace o}{E}}_{\text {in }}$ & $\begin{array}{l}\sigma \\
\dot{m} \\
\overline{1} \\
\dot{0} \\
\dot{0} \\
\stackrel{0}{\sigma}\end{array}$ & $\begin{array}{l}\bar{\sigma} \\
\dot{m} \\
\overline{1} \\
\dot{m} \\
0 \\
0 \\
\infty\end{array}$ & $\begin{array}{l}0 \\
\dot{0} \\
0 \\
0 \\
\dot{d} \\
0 \\
\dot{m}\end{array}$ & $\begin{array}{l}\sigma \\
\frac{0}{1} \\
0 \\
0 \\
0 \\
0 \\
0\end{array}$ & $\begin{array}{l}\sigma \\
\frac{\sigma}{1} \\
\vdots \\
o \\
0 \\
\vdots \\
0\end{array}$ & 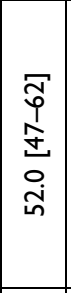 & 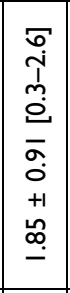 & $\begin{array}{l}0 \\
0 \\
i \\
1 \\
0 \\
0 \\
0 \\
0 \\
0 \\
0 \\
+1 \\
\infty \\
\infty \\
0 \\
-1\end{array}$ & 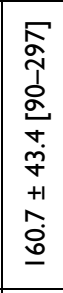 & 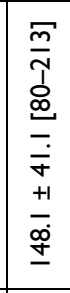 & 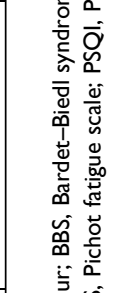 \\
\hline & 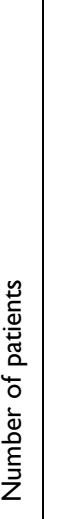 & 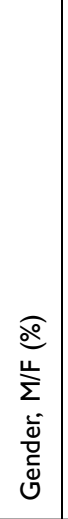 & 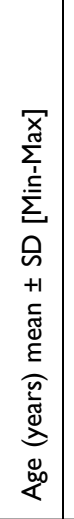 & 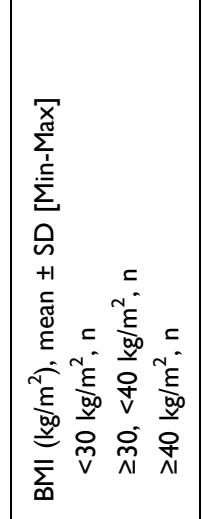 & 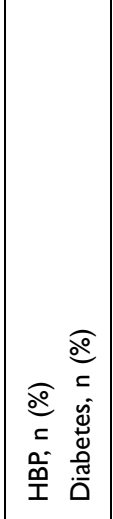 & 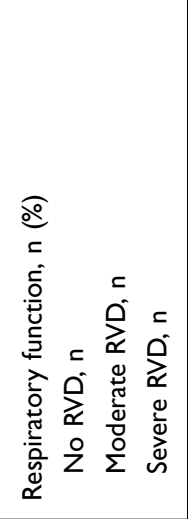 & 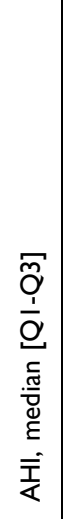 & $\begin{array}{l}\widehat{o} \\
\mathrm{~d} \\
\hat{\mathrm{v}} \\
\mathrm{v} \\
\overline{\mathrm{T}}\end{array}$ & 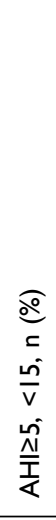 & 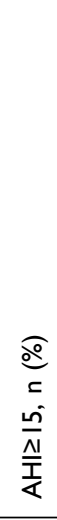 & 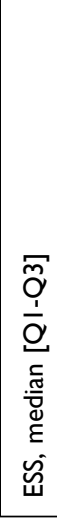 & 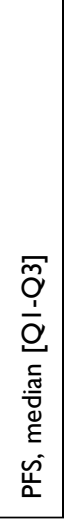 & 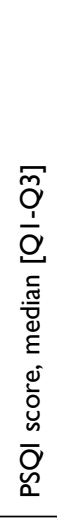 & 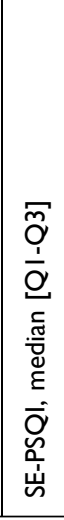 & 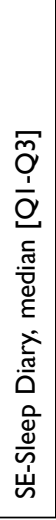 & 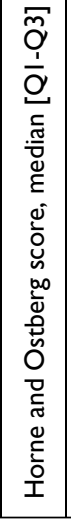 & 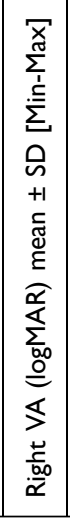 & 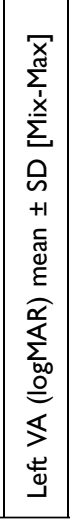 & 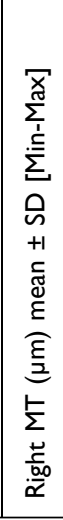 & 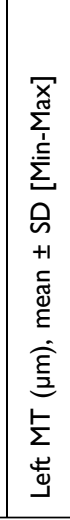 & 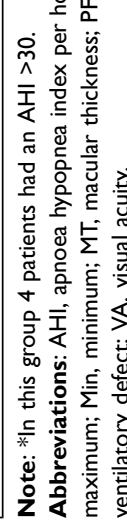 \\
\hline
\end{tabular}




\section{Effect of Sleep Apnea Syndrome, Circadian Chronotype and Vision Impairment on Quality of Sleep}

In the five patients $(17 \%)$ presenting with moderate to severe SAS quality of sleep and sleep efficacy were good (median PSQI of 2.5 [1.8-3.2] and SE-PSQI of 1.0 [1.0 1.0]). Also, in these patients, no significant daytime sleepiness (median ESS of 10.0 [8.8-12.0]) or fatigue (median PFS of 4.0 [2.8-7.0]) were found. In the same way, the 15 patients presenting with ESS and/or PFS superior to 10 had a median AHI $<5$ (ie, 2.8 [0.6-5.6] events/h), while the six patients with ESS and/or PFS superior to 15 had a median AHI of 1.5 [0-2.1] events/h. Median AHI among the 4 patients with a low SE-PSQI $(\leq 0.85)$ was normal $(1.5$ [0.8-1.8] events/h).

Regarding the eight patients reporting a poor quality of sleep (PSQI >5/21), median AHI reflected absence of SAS (1.9 [1.1-3.9] events/h) and percentage of nighttime with $\mathrm{SaO}_{2} \leq 90 \%$ was of $0.0[0.0-0.1] \%$. These results were not different as compared to patients with good quality of sleep (PSQI $\leq 5 / 21$ ) (ie, 4.3 [2.5-14.8] events/h and 0.1 [0.0-0.3] $\%$, respectively). MEQ, BCVA and CMT measurements were not significantly different between both groups.

\section{Discussion}

Moderate and severe SAS were diagnosed in 5/30 (17\%) adult BBS patients using respiratory polygraphy. One patient had predominantly central SAS without identified underlying etiology. To our knowledge, this is the first description of a central SAS in a BBS patient. Given the young age of our study population, prevalence of SAS seems relatively high. According to the data from the literature, prevalence of obstructive SAS with AHI $\geq 15$ in the general population for a comparable age group ranges from $6 \%$ to $17 \% .{ }^{17}$ Obstructive SAS was comorbid with obesity, HBP and type 2 diabetes. The prevalence of obesity was of $60 \%$ in our population (19/32). This factor is reported to have complex interactions with obstructive $\mathrm{SAS},{ }^{18}$ and to be one of its major risk factors. ${ }^{19}$ Obesity is of major concern in BBS ciliopathy and remains difficult to treat. A hypothalamic dysfunction has been described due to impaired leptin receptor trafficking associated with leptin resistance and leading to impaired body weight regulation, hyperphagia and insatiable hunger. ${ }^{20,21}$ Additional underlying risk factors specific to BBS such as hypotonia or orofacial dysmorphias should also be considered. $^{5,22}$ In a retrospective chart review, sleep disordered breathing has been clinically diagnosed in 18/ 20 BBS children. ${ }^{23}$ In this review, children's median age was of 69 months and polysomnography was performed in 10 children confirming obstructive SAS in $5(25 \%) .{ }^{23}$ In the present adult cohort, SAS was not associated with poor quality of sleep, fatigue or daytime sleepiness. We hypothesize that in some of our patients the burden due to BBS, its major visual handicap and its impact on social life, might have masked impaired sleep and daytime symptoms or participated to an altered perception. Clinical presentations of patients with obstructive SAS are heterogenous and missing complaints of insomnia and excessive daytime sleepiness have been described in different clusters of patients. ${ }^{11}$

Despite their major visual impairment due to severe pigmentary retinopathy, none of the studied patients presented with CRSWD on sleep diary. A late chronotype according to MEQ was present in only two patients. Freerunning rhythms and late chronotypes have been largely reported in totally blind people. ${ }^{10}$ Non 24-h sleep-wake cycles were mainly assessed in these studies through melatonin and cortisol secretion profiles, which we did not perform in our study. ${ }^{24,25}$ Possibly, sleep diary and MEQ alone might not be accurate enough to detect modifications of circadian rhythms in BBS patients. Usually, blinds who complain of poor sleep quality are those who completely lost the perception of light. ${ }^{26,27}$ In our study, all patients but one could either see light, hand movements or even big optotypes on at least one eye. Some persistence of light perception and the absence of CRSWD in our BBS patients suggest underlying intact photic pathways to the hypothalamus sufficient to entrain the central clock. This could also contribute to the overall rather satisfying subjective assessments on quality of sleep, sleepiness and fatigue in our patients. Moreover, BBS retinopathy is characterized by dystrophy of rods and cones. No impairment of the intrinsically photosensitive retinal ganglion cells (ipRGCs) containing the photopigment melanopsin have been reported. ${ }^{3}$ IpRGCs play a major role not only for conveying light information to the circadian clock but also in the direct effects of light on sleep and wakefulness. ${ }^{28}$ However, further explorations on the integrity of ipRGCs in BBS patients are needed.

BBS patients were not all satisfied with their sleep as poor sleep quality was reported by $37 \%$ of them (PSQI $>5 /$ 21). This finding could not be related to any specific comorbidity that we studied. Although mood disorders were not studied and might be affected, further in-depths 
investigations are required to explain this finding. Apart from circadian rhythm abnormalities, other conditions including worry, depression and painful physical disorders are also responsible of sleep disorders in blind people. ${ }^{9}$ Concerning BBS patients, particular psychological, neurodevelopmental aspects and systemic comorbidities could interfere with their perception of sleep quality, daytime vigilance and fatigue. The influence of these parameters might be difficult to discriminate.

Finally, in our study, one might question the reliability of the subjective ratings as questionnaires had to be read aloud to the patients and answers written down (N.G.). Still, assessments seemed satisfying as final scores from different evaluations were concordant in each patient.

\section{Conclusion}

Sleep apnea syndrome (SAS) was highly prevalent in this cohort of patients with Bardet-Biedl syndrome (BBS), a rare ciliopathy. Despite this finding, limited impairment of sleep quality and daytime vigilance was reported. Considering the high prevalence of cardiovascular comorbidities in BBS patients, systematic screening for sleep disordered breathing should be performed regardless of sleep and daytime vigilance complaints. Also, no impairment of circadian sleep-wake rhythms was found in these highly visually impaired patients, suggesting preserved synchronization to entrain the central clock. Further objective assessments using actigraphy and biomarkers are needed to better characterize sleep and circadian rhythms in BBS patients.

\section{Acknowledgments}

The authors would like to greatly thank the patients and everyone on the clinical and administrative teams for their assistance with this study, particularly Mrs Nathalie Goetz (organization of evaluations and collecting data) and Mrs Bernadette Kowalski for scoring the respiratory polygraphies.

\section{Author Contributions}

We declare that all authors of this manuscript have agreed on the journal to which the article will be submitted, reviewed and agreed on this version of the article before submission, agree to take responsibility and be accountable for the contents of the article. Lea Dormegny and Elisabeth Ruppert worked on the acquisition of data, the analysis and interpretation and have drafted and written the manuscript. Reana Velizarova worked on the draft of the manuscript as well as the acquisition of the data and analysis. Ülker Kilic-Huck and Henri Comtet both worked on the data analysis and critically reviewed the paper. Carmen M Schröder, Hélène Dollfus and Patrice Bourgin worked on the conception and study design, as well as the revision and critical reviewing of the article.

\section{Funding}

This study was funded by the National Hospital Program for Clinical Research (PHRC National) Bardet Biedl 2007 IDRCB 2007-A00868-45.

\section{Disclosure}

The authors declare that they have no competing interests.

\section{References}

1. Forsythe E, Beales PL. Bardet-Biedl syndrome. Eur J Hum Genet. 2013;21(1):8-13. doi:10.1038/ejhg.2012.115

2. Delvallee $C$, Nicaise $S$, Antin $M$, et al. A BBS1 SVA $\mathrm{F}$ retrotransposon insertion is a frequent cause of Bardet-Biedl syndrome. Clin Genet. 2021;99(2):318-324. doi:10.1111/cge.13878

3. Beales PL, Elcioglu N, Woolf AS, Parker D, Flinter FA. New criteria for improved diagnosis of Bardet-Biedl syndrome: results of a population survey. J Med Genet. 1999;36(6):437-446.

4. Imhoff O, Marion V, Stoetzel C, et al. Bardet-Biedl syndrome: a study of the renal and cardiovascular phenotypes in a French cohort. Clin J Am Soc Nephrol. 2011;6(1):22-29. doi:10.2215/ CJN.03320410

5. Tsang SH, Aycinena ARP, Sharma T. Ciliopathy: Bardet-Biedl syndrome. Adv Exp Med Biol. 2018;1085:171-174. doi:10.1007/ 978-3-319-95046-4_33

6. Branfield Day L, Quammie C, Heon E, et al. Liver anomalies as a phenotype parameter of Bardet-Biedl syndrome. Clin Genet. 2016;89(4):507-509. doi:10.1111/cge.12684

7. Suspitsin EN, Imyanitov EN. Bardet-Biedl syndrome. Mol Syndromol. 2016;7(2):62-71. doi:10.1159/000445491

8. Pomeroy J, VanWormer JJ, Meilahn JR, Maki T, Murali HR, Haws RM. Sleep and physical activity patterns in adults and children with Bardet-Biedl syndrome. Orphanet J Rare Dis. 2021;16(1):276. doi:10.1186/s13023-021-01911-4

9. Sack RL, Lewy AJ. Circadian rhythm sleep disorders: lessons from the blind. Sleep Med Rev. 2001;5(3):189-206. doi:10.1053/ smrv.2000.0147

10. Lockley SW, Dressman MA, Licamele L, et al. Tasimelteon for non-24-hour sleep-wake disorder in totally blind people (SET and RESET): two multicentre, randomised, double-masked, placebo-controlled Phase 3 trials. Lancet. 2015;386 (10005):1754-1764. doi:10.1016/S0140-6736(15)60031-9

11. Gagnadoux F, Le Vaillant M, Paris A, et al. Relationship between OSA clinical phenotypes and CPAP treatment outcomes. Chest. 2016;149(1):288-290. doi:10.1016/j.chest.2015.09.032

12. AASM. The International Classification of Sleep Disorders: Diagnostic \& Coding Manual (ICSD-3). 3rd ed. Darien, IL: American Academy of Sleep Medicine; 2014.

13. Buysse DJ, Reynolds CF 3rd, Monk TH, Berman SR, Kupfer DJ. The Pittsburgh Sleep Quality Index: a new instrument for psychiatric practice and research. Psychiatry Res. 1989;28(2):193-213. doi:10.1016/0165-1781(89)90047-4 
14. Johns MW. A new method for measuring daytime sleepiness: the Epworth sleepiness scale. Sleep. 1991;14(6):540-545. doi:10.1093/ sleep/14.6.540

15. Pichot P, Brun JP. Questionnaire bref d'auto-evaluation des dimensions depressive, asthenique et anxieuse [Brief self-evaluation questionnaire for depressive, asthenic and anxious dimensions]. Ann Med Psychol (Paris). 1984;142(6):862-865.

16. Horne JA, Ostberg O. A self-assessment questionnaire to determine morningness-eveningness in human circadian rhythms. Int J Chronobiol. 1976;4(2):97-110.

17. Senaratna CV, Perret JL, Lodge CJ, et al. Prevalence of obstructive sleep apnea in the general population: a systematic review. Sleep Med Rev. 2017;34:70-81. doi:10.1016/j.smrv.2016.07.002

18. Ong CW, O'Driscoll DM, Truby H, Naughton MT, Hamilton GS. The reciprocal interaction between obesity and obstructive sleep apnoea. Sleep Med Rev. 2013;17(2):123-131. doi:10.1016/j.smrv.2012.05.002

19. Tuomilehto H, Seppa J, Uusitupa M. Obesity and obstructive sleep apnea-clinical significance of weight loss. Sleep Med Rev. 2013;17 (5):321-329. doi:10.1016/j.smrv.2012.08.002

20. Feuillan PP, Ng D, Han JC, et al. Patients with Bardet-Biedl syndrome have hyperleptinemia suggestive of leptin resistance. J Clin Endocrinol Metab. 2011;96(3):E528-35. doi:10.1210/jc.2010-2290

21. Haws R, Brady S, Davis E, et al. Effect of setmelanotide, a melanocortin-4 receptor agonist, on obesity in Bardet-Biedl syndrome. Diabetes Obes Metab. 2020;22(11):2133-2140. doi:10.1111/dom. 14133
22. Panny A, Glurich I, Haws RM, Acharya A. Oral and craniofacial anomalies of Bardet-Biedl syndrome: dental management in the context of a rare disease. $J$ Dent Res. 2017;96(12):1361-1369. doi:10.1177/0022034517716913

23. Yeung JC, Katwa UA, Lee GS. Sleep disordered breathing in Bardet-Biedl syndrome. Int $J$ Pediatr Otorhinolaryngol. 2017;102:127-132. doi:10.1016/j.ijporl.2017.09.004

24. Lewy AJ, Newsome DA. Different types of melatonin circadian secretory rhythms in some blind subjects. J Clin Endocrinol Metab. 1983;56(6):1103-1107. doi:10.1210/jcem-56-6-1103

25. Arendt J, Aldhous M, Wright J. Synchronisation of a disturbed sleep-wake cycle in a blind man by melatonin treatment. Lancet. 1988;1(8588):772-773. doi:10.1016/s0140-6736(88)91586-3

26. Tabandeh H, Lockley SW, Buttery R, et al. Disturbance of sleep in blindness. Am J Ophthalmol. 1998;126(5):707-712. doi:10.1016/ s0002-9394(98)00133-0

27. Leger DG, Paillard C. Epidemiology of sleep disorders in blind people. Sleep Res. 1995;23:527.

28. Hubbard J, Kobayashi Frisk M, Ruppert E, et al. Dissecting and modeling photic and melanopsin effects to predict sleep disturbances induced by irregular light exposure in mice. Proc Natl Acad Sci USA. 2021;118(25). doi:10.1073/pnas.2017364118

\section{Publish your work in this journal}

Nature and Science of Sleep is an international, peer-reviewed, open access journal covering all aspects of sleep science and sleep medicine, including the neurophysiology and functions of sleep, the genetics of sleep, sleep and society, biological rhythms, dreaming, sleep disorders and therapy, and strategies to optimize healthy sleep.
The manuscript management system is completely online and includes a very quick and fair peer-review system, which is all easy to use. Visit http://www.dovepress.com/testimonials.php to read real quotes from published authors. 